\title{
Optimum MIMO-OFDM receivers with imperfect channel state information*
}

\author{
Giulio Coluccia \\ Politecnico di Torino, Italy \\ Erwin Riegler \\ $\mathrm{ftw}$, Austria \\ Email: giulio.coluccia@polito.it E-mail: riegler@ftw.at
}

\author{
Christoph Mecklenbrauker \\ Vienna University of Technology \\ Email: cfm@nt.tuwien.ac.at
}

\author{
Giorgio Taricco \\ Politecnico di Torino, Italy \\ Email: giorgio.taricco@polito.it
}

\begin{abstract}
Channel estimation inaccuracy is known to affect significantly the error performance of coded communication systems. This applies in particular to broadband MIMO channels, often considered in conjunction with OFDM such as in the IEEE 802.11n and 802.16 standards. The focus of this work is on an 802.11n compliant MIMO-OFDM communication system. Different channel estimation techniques (based on pilot symbol insertion) are considered and their relevant error performance is analyzed. More specifically, genie-aided, mismatched, and optimum channel estimation techniques are studied with special emphasis on the last one as far as concerns the relative error performance versus complexity trade-off in suboptimum implementation. It is shown that the optimum receiver can be implemented by limiting the channel processing to the dominant eigenmodes, in order to reduce the ensuing complexity. The approach followed in this work may be seen as an extension of previous results relevant to the narrowband MIMO channel.
\end{abstract}

\section{INTRODUCTION}

Channel estimation is a key issue to the implementation of digital receivers. In most cases, it is assumed to be perfectly available but this is rarely the case as it is usually obtained by pilot symbol insertion and may be affected by significant inaccuracies. In many cases, obtaining accurate channel state information (CSI) requires to devote a substantial amount of resources to this end and may reduce significantly the efficiency of a communication system.

This problem was recently addressed in the case of narrowband MIMO communications and optimum receiver schemes have been designed for the uncorrelated Rayleigh fading for the correlated Rician fading cases in [1] and [2], respectively. These works proposed optimum receiver schemes implementing joint channel estimation and decoding by the use of pilot symbol insertion and space-time trellis codes, without explicitly estimating the channel matrix.

A key assumption in [2] is the exact knowledge of the channel distribution at the receiver. More precisely, separately correlated (Kronecker) Rician fading has been considered here [3]. Although this model is known to over-simplify the actual covariance structure of the MIMO channel [4], the receiver was shown to perform very well in simulations based on measured channels in real environments [5].

\footnotetext{
* This work is supported by the STREP project No. IST-026905 (MASCOT) within the sixth framework programme of the European Commission.

The work of Giorgio Taricco has been supported by the EC-funded Network of Excellence NEWCOM++.
}

Here, we consider a MIMO-OFDM communication system and extend the optimum space-time decoder to frequencyselective slow Rician fading channel with multiple transmit and receive antennas. The blocks that compose the transmitter are compliant with the $802.11 \mathrm{n}$ draft 2.0 standard [6]. The proposed receiver is based on a wideband block fading MIMO-OFDM channel matrix model which is completely characterized by its first- and second-order statistics. The channel matrix model allows for spatial correlation at the transmitter and receiver side, as well as for spectral correlation between the subcarriers. However, we strongly restrict the covariance structure of the MIMO-OFDM channel model to the Kronecker product of these individual covariances.

\section{A. Notation and Definitions}

We denote (column-) vectors and matrices by lowercase and uppercase boldface characters, respectively. The $n$th element of a vector $\mathbf{x}$ is $(\mathbf{x})_{n}$. The $(m, n)$ th element of a matrix $\mathbf{A}$ is $(\mathbf{A})_{m n}$. The $n$th row of $\mathbf{A}$ is $(\mathbf{A})_{n}$. The transpose (Hermitian transpose) of $\mathbf{A}$ is $\mathbf{A}^{\top}\left(\mathbf{A}^{\mathrm{H}}\right)$. The (exponential of the) trace of $\mathbf{A}$ is $(\operatorname{etr}(\mathbf{A})) \operatorname{Tr}(\mathbf{A})$. The square Frobenius norm of $\mathbf{A}$ can be written as $\|\mathbf{A}\|^{2}=\operatorname{Tr}\left(\mathbf{A A}^{\mathbf{H}}\right)$. The notation $\mathbf{A} \otimes \mathbf{B}$ denotes the Kronecker product of $\mathbf{A}$ times $\mathbf{B}$. The notation $\mathbf{A} \oplus \mathbf{B}=\operatorname{diag}(\mathbf{A}, \mathbf{B})$ denotes the matrix direct sum [7] of A plus $\mathbf{B}$. The notation vec $\{\mathbf{A}\}$ denotes the column vector obtained by stacking the columns of $\mathbf{A}$ on top of each other from left to right. The matrix $\mathbf{A}^{1 / 2}$ is the matrix square-root of the Hermitian nonnegative definite matrix $\mathbf{A}$. The notation $\mathbb{E}_{\mathbf{H}}[(\cdot)]$ represents the expectation of (.) with respect to the random matrix $\mathbf{H}$. The notation $\mathbf{x} \sim \mathcal{N}_{c}\left(\overline{\mathbf{x}}, \mathbf{R}_{x}\right)$ means that the complex random column vector $\mathbf{x}$ is circularly-symmetric Gaussian distributed, its mean is $\overline{\mathbf{x}}=\mathbb{E}[\mathbf{x}]$, its covariance matrix is $\mathbf{R}_{x}=\mathbb{E}\left[(\mathbf{x}-\overline{\mathbf{x}})(\mathbf{x}-\overline{\mathbf{x}})^{\mathrm{H}}\right]$, and its probability density function is given by

$$
\begin{gathered}
p(\mathbf{x})=\operatorname{det}\left(\pi \mathbf{R}_{x}\right)^{-1} \exp \left(-(\mathbf{x}-\overline{\mathbf{x}})^{\mathrm{H}} \mathbf{R}_{x}^{-1}(\mathbf{x}-\overline{\mathbf{x}})\right) . \\
\text { II. SYSTEM MODEL }
\end{gathered}
$$

We consider a single-user wideband MIMO-OFDM block fading channel with $n_{\mathrm{F}}$ subcarriers, $n_{\mathrm{T}}$ transmit and $n_{\mathrm{R}}$ receive antennas. A frame is composed by $P$ pilot and $N$ data symbol intervals. Thus, during each frame and for each subcarrier, the transmitter sends a matrix $\mathbf{X}_{\mathrm{P}, k} \in \mathbb{C}^{n_{\mathrm{T}} \times P}$ of pilot symbols 
$\left(k=1, \ldots, n_{\mathrm{F}}\right)$ followed by a matrix $\mathbf{X}_{k} \in \mathbb{C}^{n_{\mathrm{T}} \times N}$ of data symbols.

The global input-output relation, after Cyclic Prefix removal, can be written as

$$
\mathbf{Y}_{\mathrm{P}}=\mathbf{H X}_{\mathrm{P}}+\mathbf{Z}_{\mathrm{P}}, \quad \mathbf{Y}=\mathbf{H X}+\mathbf{Z}
$$

where $\mathbf{X} \in \mathbb{C}^{n_{\mathrm{T}} n_{\mathrm{F}} \times N}$ and $\mathbf{X}_{\mathrm{P}} \in \mathbb{C}^{n_{\mathrm{T}} n_{\mathrm{F}} \times P}$ denote the block matrices obtained by stacking on top of each other $\mathbf{X}_{k}$ and $\mathbf{X}_{\mathrm{P}, k}$, respectively. Here, $\mathbf{H}$ is an $n_{\mathrm{R}} n_{\mathrm{F}} \times n_{\top} n_{\mathrm{F}}$ block diagonal channel matrix whose characteristics will be discussed in Section II-A. $\mathbf{Z}_{\mathrm{P}} \in \mathbb{C}^{n_{\mathrm{R}} n_{\mathrm{F}} \times P}$ and $\mathbf{Z} \in \mathbb{C}^{n_{\mathrm{R}} n_{\mathrm{F}} \times N}$ are two matrices representing the receiver noise with independent circularly-symmetric zero-mean complex Gaussian entries of variance $N_{0}$ (Here we assume that the noise affecting different frequencies is uncorrelated to each other [8, Section II.B]).

\section{A. Channel Matrix}

We extend the classic narrowband Kronecker model to the frequency domain. The channel matrix $\mathbf{H}$ is a block diagonal matrix

$$
\mathbf{H} \triangleq \bigoplus_{k=1}^{n_{\mathrm{F}}} \mathbf{H}_{k}=\operatorname{diag}\left(\mathbf{H}_{1}, \ldots, \mathbf{H}_{n_{\mathrm{F}}}\right)
$$

where for each subcarrier the following properties hold:

$$
\mathbf{H}_{k} \triangleq \overline{\mathbf{H}}_{k}+\sqrt{\frac{1}{K_{k}+1}}\left(\left(\mathbf{F}^{1 / 2}\right)_{k} \otimes \mathbf{R}^{1 / 2}\right) \mathbf{W} \mathbf{T}^{1 / 2}
$$

The $n_{\mathrm{R}} \times n_{\mathrm{R}}$ matrix $\mathbf{R}$ and the $n_{\mathrm{T}} \times n_{\mathrm{T}}$ matrix $\mathbf{T}$ are the receive and transmit correlation matrices, respectively. The $n_{\mathrm{F}} \times n_{\mathrm{F}}$ matrix $\mathbf{F}$ is the frequency carriers correlation matrix. $\mathbf{R}, \mathbf{T}$ and $\mathbf{F}$ are assumed to be Hermitian and positive definite. $\mathbf{W}$ is a $n_{\mathrm{R}} n_{\mathrm{F}} \times n_{\mathrm{\top}}$ matrix containing independent circularly-symmetric zero-mean complex Gaussian entries with unit variance. $\overline{\mathbf{H}}_{k}$ is the matrix of LOS components at a given frequency $k$ and it is normalized in order to have squared Frobenius norm $\left\|\overline{\mathbf{H}}_{k}\right\|^{2}=\frac{K_{k}}{K_{k}+1} n_{\mathrm{\top}} n_{\mathrm{R}} . K_{k}$ is the Rice factor related to frequency $k$, which represents the relative strength of the LOS component at frequency $k$. Following the definition in [8], we can see that

$$
K_{k}=\frac{\left\|\overline{\mathbf{H}}_{k}\right\|^{2}}{\mathbb{E}\left[\left\|\mathbf{H}_{k}-\overline{\mathbf{H}}_{k}\right\|^{2}\right]}
$$

Thus, we have, independently of $K_{k}$,

$$
\mathbb{E}\left[\operatorname{Tr}\left(\mathbf{H}_{k} \mathbf{H}_{k}^{\mathrm{H}}\right)\right]=n_{\mathrm{T}} n_{\mathrm{R}} .
$$

It can be shown that according to this model the covariance of the entries of $\mathbf{H}$ is given in terms of a product of the entries of the correlation matrices $\mathbf{R}, \mathbf{T}$ and $\mathbf{F}$. Namely, we have:

$$
\operatorname{cov}\left(\left(\mathbf{H}_{k}\right)_{i j},\left(\mathbf{H}_{k^{\prime}}\right)_{i^{\prime} j^{\prime}}\right)=\frac{\mathbf{R}_{i i^{\prime}} \mathbf{T}_{j j^{\prime}}^{*} \mathbf{F}_{k k^{\prime}}}{\sqrt{\left(K_{k}+1\right)\left(K_{k^{\prime}}+1\right)}}
$$

where $\operatorname{cov}(X, Y) \triangleq \mathbb{E}\left[X Y^{*}\right]-\mathbb{E}[X] \mathbb{E}[Y]^{*}$.

\section{RECEIVER ARCHITECTURE}

The receivers we design in the following are not based on the classical assumption of perfect channel state information at the receiver (CSIR): they estimate the CSIR by the means of pilot symbols insertion. The Genie Receiver is an ideal receiver equipped with a perfect CSIR. The Mismatched receiver first estimates the CSIR, then uses the estimate to decode the symbol by a perfect CSIR approach. The Optimum receiver does not explicitly estimate the CSIR, but jointly performs channel estimation and symbol decoding. Since the channel coding is assumed to be performed across frequency dimension, for each receiver architecture we propose an iterative formulation suitable to Viterbi decoding.

\section{A. Genie Receiver}

The ideal genie-aided receiver (considered here as a lower bound on FER) output is given by

$$
\widehat{\mathbf{X}}_{\mathrm{GR}}=\arg \min _{\mathbf{X}} \mu_{\mathrm{GR}}(\mathbf{X} \mid \mathbf{Y})
$$

where

$$
\mu_{\mathrm{GR}}(\mathbf{X} \mid \mathbf{Y})=\|\mathbf{Y}-\mathbf{H X}\|^{2}=\sum_{k=1}^{n_{\mathrm{F}}}\left\|\mathbf{Y}_{k}-\mathbf{H}_{k} \mathbf{X}_{k}\right\|^{2}
$$

\section{B. Mismatched Receiver}

We follow the approach of [1], [2] to obtain a maximumlikelihood (ML) ${ }^{1}$ estimation of $\mathbf{H}_{k}$ :

$$
\widehat{\mathbf{H}}_{k}=\mathbf{Y}_{\mathrm{P}, k} \mathbf{X}_{\mathrm{P}, k}^{\mathrm{H}}\left(\mathbf{X}_{\mathrm{P}, k} \mathbf{X}_{\mathrm{P}, k}^{\mathrm{H}}\right)^{-1}
$$

provided that $\left(\mathbf{X}_{\mathrm{P}, k} \mathbf{X}_{\mathrm{P}, k}^{\mathrm{H}}\right)$ is invertible. Then the mismatched receiver output is given by

$$
\widehat{\mathbf{X}}_{\mathrm{MR}}=\arg \min _{\mathbf{X}} \mu_{\mathrm{MR}}(\mathbf{X} \mid \mathbf{Y})
$$

where

$$
\mu_{\mathrm{MR}}(\mathbf{X} \mid \mathbf{Y})=\sum_{k=1}^{n_{\mathrm{F}}}\left\|\mathbf{Y}_{k}-\widehat{\mathbf{H}}_{k} \mathbf{X}_{k}\right\|^{2}
$$

\section{Optimum Receiver}

The optimum receiver output is given by

$$
\widehat{\mathbf{X}}_{\mathrm{OR}}=\arg \min _{\mathbf{X}} \mu_{\mathrm{OR}}\left(\mathbf{X} \mid \mathbf{Y}, \mathbf{Y}_{\mathrm{P}}, \mathbf{X}_{\mathrm{P}}\right)
$$

where

$$
\mu_{\mathrm{OR}}\left(\mathbf{X} \mid \mathbf{Y}, \mathbf{Y}_{\mathrm{P}}, \mathbf{X}_{\mathrm{P}}\right)=-\ln \operatorname{Pr}\left(\mathbf{X} \mid \mathbf{X}_{p}, \mathbf{Y}, \mathbf{Y}_{p}\right)
$$

Assuming equally likely transmitted words and the conditional independence of $\mathbf{Y}$ and $\mathbf{Y}_{p}$ given $\mathbf{X}, \mathbf{H}$ and $\mathbf{X}_{p}, \mathbf{H}$, respectively, the probability in (10) can be written as

$$
\begin{aligned}
\operatorname{Pr}\left(\mathbf{X} \mid \mathbf{X}_{p}, \mathbf{Y}, \mathbf{Y}_{p}\right) & =\mathbb{E}_{\mathbf{H}}\left[p\left(\mathbf{Y}, \mathbf{Y}_{p} \mid \mathbf{X}, \mathbf{X}_{p}, \mathbf{H}\right)\right] \\
& =\mathbb{E}_{\mathbf{H}}\left[p(\mathbf{Y} \mid \mathbf{X}, \mathbf{H}) p\left(\mathbf{Y}_{p} \mid \mathbf{X}_{p}, \mathbf{H}\right)\right]
\end{aligned}
$$

${ }^{1} \mathrm{~A}$ minimum-mean-square-error (MMSE) estimation can be also obtained. In [2] it is demonstrated that ML and MMSE estimates tend to each other when SNR grows large 
Then, the a posteriori probability (11) can be calculated by writing explicitly the probability density functions involved. After some algebra we obtain

$$
\begin{aligned}
& p\left(\mathbf{Y}, \mathbf{Y}_{p} \mid \mathbf{X}, \mathbf{X}_{p}, \mathbf{H}\right)=\left(\pi N_{0}\right)^{-(N+P) n_{\mathrm{R}} n_{\mathrm{F}}} \\
& \cdot \exp \left(-\frac{\left\|\mathbf{Y}_{\mathbf{P}}-\overline{\mathbf{H}} \mathbf{X}_{\mathbf{P}}\right\|^{2}+\|\mathbf{Y}-\overline{\mathbf{H}} \mathbf{X}\|^{2}}{N_{0}}\right) \\
& \cdot \exp \left(-\mathbf{w}^{\mathrm{H}} \mathbf{C} \mathbf{w}+\mathbf{b}^{\mathrm{H}} \mathbf{w}+\mathbf{w}^{\mathrm{H}} \mathbf{b}\right)
\end{aligned}
$$

where we defined

$$
\left\{\begin{array}{l}
\overline{\mathbf{H}} \triangleq \bigoplus_{k=1}^{n_{\mathrm{F}}} \overline{\mathbf{H}}_{k} \\
\mathbf{w} \triangleq \operatorname{vec}\{\mathbf{W}\} \\
\mathbf{b} \triangleq \sum_{k=1}^{n_{\mathrm{F}}} \operatorname{vec}\left\{\left[\left(\mathbf{F}^{1 / 2}\right)_{k} \otimes \mathbf{R}^{1 / 2}\right]^{\mathrm{H}} \mathbf{B}_{k} \mathbf{T}^{1 / 2}\right\} / N_{0} \\
\mathbf{B}_{k} \triangleq \frac{\left(\mathbf{Y}_{k}-\overline{\mathbf{H}}_{k} \mathbf{X}_{k}\right) \mathbf{X}_{k}^{\mathrm{H}}+\left(\mathbf{Y}_{\mathrm{P}, k}-\overline{\mathbf{H}}_{k} \mathbf{X}_{\mathrm{P}, k}\right) \mathbf{X}_{\mathrm{P}, k}^{\mathrm{H}}}{\sqrt{K_{k}+1}} \\
\mathbf{C} \triangleq \sum_{k=1}^{n_{\mathrm{F}}} \mathbf{A}_{k}^{\mathrm{T}} \otimes\left[\left(\mathbf{F}^{1 / 2}\right)_{k} \otimes \mathbf{R}^{1 / 2}\right]^{\mathrm{H}}\left[\left(\mathbf{F}^{1 / 2}\right)_{k} \otimes \mathbf{R}^{1 / 2}\right] \\
\mathbf{A}_{k} \triangleq \frac{\mathbf{T}^{1 / 2}\left(\mathbf{X}_{k} \mathbf{X}_{k}^{\mathrm{H}}+\mathbf{X}_{\mathrm{P}, k} \mathbf{X}_{\mathrm{P}, k}^{\mathrm{H}}\right) \mathbf{T}^{1 / 2}}{N_{0}\left(K_{k}+1\right)}
\end{array}\right.
$$

and we used the property (19). Calculating the expectation of (12) over w and using equation (20), we obtain the following result:

$$
\begin{aligned}
& \mathbb{E}_{\mathbf{w}}\left\{p\left(\mathbf{Y}, \mathbf{Y}_{p} \mid \mathbf{X}, \mathbf{X}_{p}, \mathbf{w}\right)\right\}=\left(\pi N_{0}\right)^{-(N+P) n_{\mathrm{R}} n_{\mathrm{F}}} \\
& \cdot \exp \left(-\frac{\left\|\mathbf{Y}_{\mathbf{P}}-\overline{\mathbf{H}} \mathbf{X}_{\mathbf{P}}\right\|^{2}+\|\mathbf{Y}-\overline{\mathbf{H}} \mathbf{X}\|^{2}}{N_{0}}\right) \\
& \cdot \operatorname{det}\left(\mathbf{I}_{n_{\mathrm{T}} n_{\mathrm{R}} n_{\mathrm{F}}}+\mathbf{C}\right)^{-1} \exp \left(\mathbf{b}^{\mathrm{H}}\left(\mathbf{I}_{n_{\top} n_{\mathrm{R}} n_{\mathrm{F}}}+\mathbf{C}\right)^{-1} \mathbf{b}\right)
\end{aligned}
$$

Hence, after proper scaling and dropping terms independent of $\mathbf{X}$, the decision metric for the optimum receiver is obtained as

$$
\begin{aligned}
& \mu_{\mathrm{OR}}\left(\mathbf{X} \mid \mathbf{Y}, \mathbf{Y}_{\mathrm{P}}, \mathbf{X}_{\mathrm{P}}\right)=\|\mathbf{Y}-\overline{\mathbf{H}} \mathbf{X}\|^{2} \\
& +N_{0} \ln \operatorname{det}\left(\mathbf{I}_{n_{\mathrm{T}} n_{\mathrm{R}} n_{\mathrm{F}}}+\mathbf{C}\right)-N_{0} \mathbf{b}^{\mathrm{H}}\left(\mathbf{I}_{n_{\mathrm{T}} n_{\mathrm{R}} n_{\mathrm{F}}}+\mathbf{C}\right)^{-1} \mathbf{b}
\end{aligned}
$$

The optimum receiver requires to know several parameters of the statistical distribution of channel matrix $\mathbf{H}$. In [2] it has been shown that these parameters can be estimated within the decoding process with almost no degradation of the system performance.

1) Iterative computation: Following now the approach of [1], [2], we resort to an iterative algorithm to reduce the computational complexity of an exhaustive decoding. The metric (14) is first evaluated along frequency dimension; then, if the codeword is longer than one OFDM symbol, the evaluation can be extended symbol by symbol. For simplicity we focus on the case of (modulated) codewords that perfectly match the OFDM symbols size $n_{\mathrm{F}}$.
We then rewrite the metric (14) as

$$
\mu_{\mathrm{OR}}\left(\mathbf{X}, \mathbf{Y}, \mathbf{X}_{p}, \mathbf{Y}_{p}\right)=\sum_{k=1}^{n_{\mathrm{F}}} \Delta \mu_{k}\left(\mathbf{X}_{k}, \mathbf{Y}_{k}, \mathbf{X}_{\mathrm{P}, k}, \mathbf{Y}_{\mathrm{P}, k}\right)
$$

where the branch metric $\Delta \mu_{k}\left(\mathbf{X}_{k}, \mathbf{Y}_{k}, \mathbf{X}_{\mathbf{P}, k}, \mathbf{Y}_{\mathrm{P}, k}\right)$ is given by

$$
\begin{aligned}
\Delta \mu_{k}\left(\mathbf{X}_{k}, \mathbf{Y}_{k}, \mathbf{X}_{\mathbf{P}, k}, \mathbf{Y}_{\mathrm{P}, k}\right)= & \left\|\mathbf{Y}_{k}-\overline{\mathbf{H}}_{k} \mathbf{X}_{k}\right\|^{2} \\
& +N_{0} \ln \operatorname{det}\left(\mathbf{I}_{n_{\top} n_{\mathrm{R}}}+\mathbf{U} \boldsymbol{\Lambda}_{-} \mathbf{U}^{\mathrm{H}}\right) \\
& -\mathbf{b}^{\mathrm{H}} \boldsymbol{\Lambda} \mathbf{b} / N_{0}+\mathbf{b}_{-}^{\mathrm{H}} \boldsymbol{\Lambda}_{-} \mathbf{b}_{-} / N_{0}
\end{aligned}
$$

Here, with the subscript $(\cdot)_{-}$we denote $(\cdot)$ calculated at the previous iteration and we define:

$$
\left\{\begin{array}{l}
\mathbf{U} \triangleq \mathbf{L}_{k} \otimes\left(\left(\mathbf{F}^{1 / 2}\right)_{k} \otimes \mathbf{R}^{1 / 2}\right) \\
\boldsymbol{\Lambda} \triangleq \boldsymbol{\Lambda}_{-}+\Delta \boldsymbol{\Lambda} \\
\Delta \boldsymbol{\Lambda} \triangleq-\boldsymbol{\Lambda}_{-} \mathbf{U}^{\mathrm{H}}\left(\mathbf{I}_{n_{\mathrm{T}} n_{\mathrm{R}}}+\mathbf{U} \boldsymbol{\Lambda}_{-} \mathbf{U}^{\mathrm{H}}\right)^{-1} \mathbf{U} \boldsymbol{\Lambda}_{-} \\
\boldsymbol{\Lambda}_{0} \triangleq \mathbf{I}_{n_{\mathrm{T}} n_{\mathrm{R}} n_{\mathrm{F}}} \\
\mathbf{b} \triangleq \mathbf{b}_{-}+\Delta \mathbf{b} \\
\Delta \mathbf{b} \triangleq \operatorname{vec}\left\{\left[\left(\mathbf{F}^{1 / 2}\right)_{k} \otimes \mathbf{R}^{1 / 2}\right]^{\mathrm{H}} \mathbf{B}_{k} \mathbf{T}^{1 / 2}\right\}
\end{array}\right.
$$

and $\mathbf{L}_{k}$ is the Cholesky decomposition of $\mathbf{A}_{k}^{\top}$ such that $\mathbf{A}_{k}^{\top}=$ $\mathbf{L}_{k}^{\mathrm{H}} \mathbf{L}_{k} . \boldsymbol{\Lambda}_{0}$ is the initialization of $\boldsymbol{\Lambda}$.

2) Eigenvalue approximation: Deriving (16) from (14) we have reduced the dimension of the matrix inversion from $n_{\mathrm{T}} n_{\mathrm{R}} n_{\mathrm{F}}$ to $n_{\mathrm{R}} n_{\mathrm{T}}$. Since the dimension $n_{\mathrm{F}}$ can assume very large values (in the example we will take into account in section IV $n_{\mathrm{F}}=117$ ), this parameter has a strong impact on the system complexity (and then performance). We further reduce the complexity of the algorithm as explained in the following.

Let $\mathbf{V}$ be an unitary matrix such that $\mathbf{V F V}^{\mathrm{H}}=\boldsymbol{\Lambda}_{\mathbf{F}}$ represents the eigenvalue decomposition of $\mathbf{F}$ and define the projection $\mathbf{P}_{m_{\mathrm{F}}}=\left(\mathbf{I}_{m_{\mathrm{F}}}, \mathbf{0}_{m_{\mathrm{F}} \times n_{\mathrm{F}}-m_{\mathrm{F}}}\right)^{\mathrm{T}}$ on the dominant eigenvalue subspace of $\mathbf{F}$ and the matrix $\widetilde{\mathbf{V}}=\mathbf{V} \mathbf{P}_{m_{\mathrm{F}}}$. Since it can be shown that the metric (14) is invariant under the transformation $\left(\mathbf{F}^{1 / 2}\right)_{k} \rightarrow\left(\mathbf{F}^{1 / 2}\right)_{k} \mathbf{V}$, then we approximate $\mathbf{F}^{1 / 2}$ by $\widetilde{\mathbf{F}}^{1 / 2} \triangleq \mathbf{F}^{1 / 2} \widetilde{\mathbf{V}}$, reducing its dimension from $n_{\mathbf{F}}$ to $m_{\mathrm{F}}$. The impact of this approximation will be discussed in section IV.

\section{NumericAl Results}

In this section we consider a MIMO-OFDM system with $n_{\mathrm{R}}=2$ receive antennas. The blocks that compose the transmitter are compliant with the 802.11n draft 2.0 standard [6]. In particular, we choose the Modulation and Coding Scheme (MCS) 1 with $40 \mathrm{MHz}$ channel (Greenfield format) and the (optional) Space-Time Block Code STBC [9]; the number of subcarriers is $n_{\mathrm{F}}=117$ (108 among which are dedicated to data ${ }^{2}$ ). The random bit stream is fed into a Binary Convolutional Encoder with standard generator polynomials,

\footnotetext{
${ }^{2}$ See [6] for the details
} 
$g_{0}=133_{8}$ and $g_{1}=171_{8}$, of rate $R=1 / 2$. Coded bits are modulated with QPSK and Gray mapping. Complex QPSK symbols are pairwise (since the presence of STBC) mapped on the data subcarriers. For every subcarrier the standard STBC maps each pair of symbols $\left(x_{k, 1} x_{k, 2}\right)$ to a $2 \times 2$ matrix

$$
\left(\begin{array}{ll}
x_{k, 1} & x_{k, 2}
\end{array}\right) \mapsto\left(\begin{array}{cc}
x_{k, 1} & x_{k, 2} \\
-x_{k, 2}^{*} & x_{k, 1}^{*}
\end{array}\right)
$$

to be transmitted by the $n_{\mathrm{T}}=2$ transmit antennas. We choose to transmit the maximum amount of bits (208 of data plus 6 of tail and 2 of padding) contained in one OFDM symbol: each subcarrier matrix $\mathbf{X}_{k}$ has length $N=2$. The data symbol matrix is preceded by a pilot symbol matrix such that $\mathbf{X}_{\mathrm{P}, k} \mathbf{X}_{\mathrm{P}, k}^{\mathrm{H}} \propto \mathbf{I}_{n_{\mathrm{T}}}$ for each $k$. For $n_{\mathrm{T}}=2$ the standard sets $P=2$. Both the data and the pilot matrices are normalized in order to transmit unitary energy at each symbol period.

Simulations have been carried out for the mismatched and the optimum receivers; the genie receiver has been considered as an ideal reference.

We consider a zero-mean channel, with correlation between subcarriers, in absence/presence of spatial correlation at the transmitter and the receiver.

Transmit and receive correlation matrices $\mathbf{T}$ and $\mathbf{R}$ are given according to the exponential model [10]:

$$
(\mathbf{T})_{i j}=\left\{\begin{array}{c}
\rho_{\mathrm{T}}^{j-i}, i \leq j \\
(\mathbf{T})_{j i}^{*}, i>j
\end{array} \quad \text { and } \quad(\mathbf{R})_{i j}=\left\{\begin{array}{c}
\rho_{\mathrm{R}}^{j-i}, i \leq j \\
(\mathbf{R})_{j i}^{*}, i>j
\end{array}\right.\right.
$$

where $\rho_{\mathrm{T}}$ and $\rho_{\mathrm{R}}$ are the complex correlation coefficients of neighboring transmit and receive antennas, respectively.

The correlation between carriers is modeled as follows [11]:

$$
(\mathbf{F})_{i j}=\left[\sqrt{1+\left(|i-j| \frac{\Delta f}{\Delta f_{c}}\right)^{2}}\right]^{-1}
$$

where $\Delta f=312.5 \mathrm{kHz}$ is the separation between carriers set by the standard and $\Delta f_{c}=10 \mathrm{MHz}$ is the coherence bandwidth of the channel.

Fig. 1 plots the square Frobenius norms of $\mathbf{F}^{1 / 2}$ and $\widetilde{\mathbf{F}}^{1 / 2}$ versus the number of eigenvalues $m_{\mathrm{F}}$. It can be noticed that only the first 8 eigenvalues contain the whole energy of $\mathbf{F}^{1 / 2}$. We then choose to study 3 cases: $i$ ) below the threshold $\left(m_{\mathrm{F}}=\right.$ $4)$; ii) in correspondence of the threshold $\left(m_{\mathrm{F}}=8\right)$; iii) beyond the threshold $\left(m_{\mathrm{F}}=16\right)$.

Performance results are reported in terms of FER (Frame Error Rate) versus SNR (Signal to Noise Ratio) in dB.

Fig. 2 shows the performance of the system when $\rho_{\mathrm{R}}=$ $\rho_{\mathrm{T}}=0.0$ (Uncorrelated case). We plot the results obtained for the Genie receiver, for the Mismatched receiver and for the proposed Optimum wideband receiver with $m_{\mathrm{F}}=4,8,16$. It can be noticed that the optimum wideband receiver attains its best performance already with $m_{\mathrm{F}}=8$; the curve with $m_{\mathrm{F}}=16$ is overlapping. The penalty with respect to the Genie Receiver is about $0.7 \mathrm{~dB}$. The use of $m_{\mathrm{F}}=4$ introduces a further degradation of about $0.5 \mathrm{~dB}$ (@ FER $=10^{-2}$ ): moreover, for $m_{\mathrm{F}}=4$ we do not only have a penalty in $\mathrm{dB}$ but also a diversity loss. The curves also show that the gain

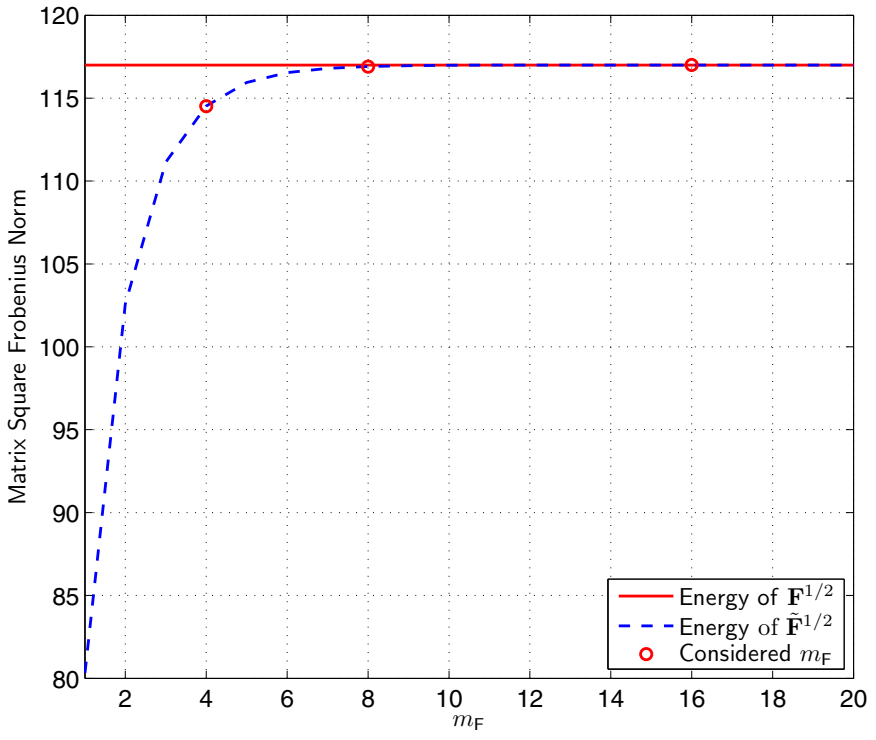

Fig. 1. Energy of $\mathbf{F}^{1 / 2}$ and $\widetilde{\mathbf{F}}^{1 / 2}$ vs. $m_{\mathrm{F}}$. The considered values of $m_{\mathrm{F}}=$ $4,8,16$ are highlighted.

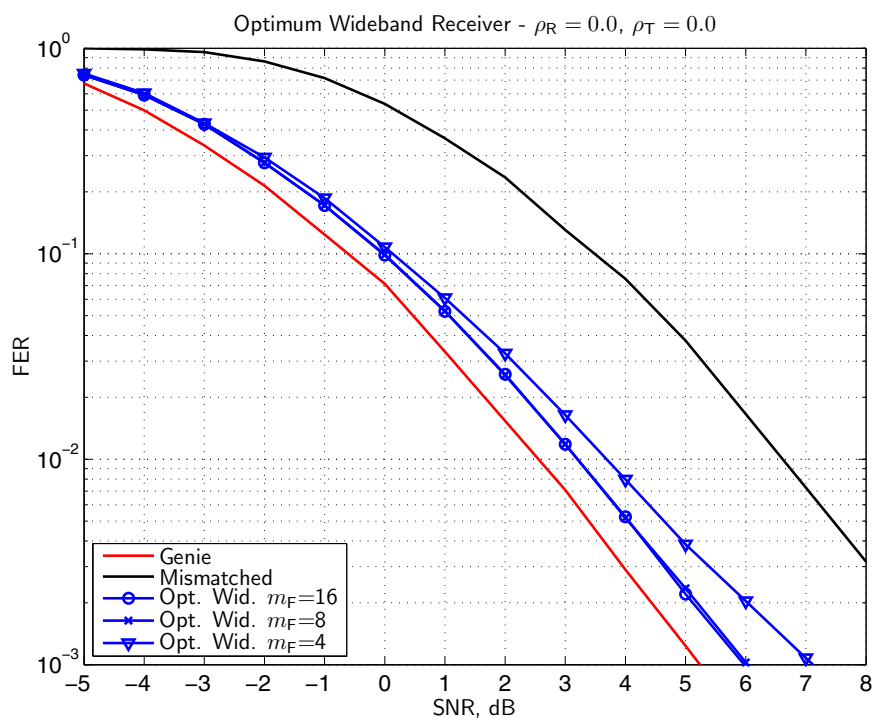

Fig. 2. FER vs. SNR of the MIMO-OFDM system with $\rho_{\mathrm{R}}=\rho_{\mathrm{T}}=0.0$. Genie, Mismatched and Optimum $\left(m_{\mathrm{F}}=4,8,16\right)$ receivers.

that can be obtained using the Optimum Receiver with respect to the standard Mismatched receiver is more than $3 \mathrm{~dB}$.

In Fig. 3 we report the performance of the same system but in a spatially-correlated environment $\left(\rho_{\mathrm{R}}=\rho_{\mathrm{T}}=0.7\right)$. It can be noticed that the spatial correlation introduces a penalty with respect to the uncorrelated case of about $2.5 \mathrm{~dB}$. The Optimum receiver with $m_{\mathrm{F}}=8,16$ loses about $0.7 \mathrm{~dB}$ from the Genie receiver, whereas using $m_{\mathrm{F}}=4$ the curves show a further penalty of $1.3 \mathrm{~dB}$ (@ FER $=10^{-2}$ ). The gain that can be obtained using the Optimum rather than the Mismatched receiver is in this case $3.5 \mathrm{~dB}$.

These results show that a substantial complexity reduction can be obtained considering $\widetilde{\mathbf{F}}^{1 / 2}$ rather than $\mathbf{F}^{1 / 2}$. In fact, 


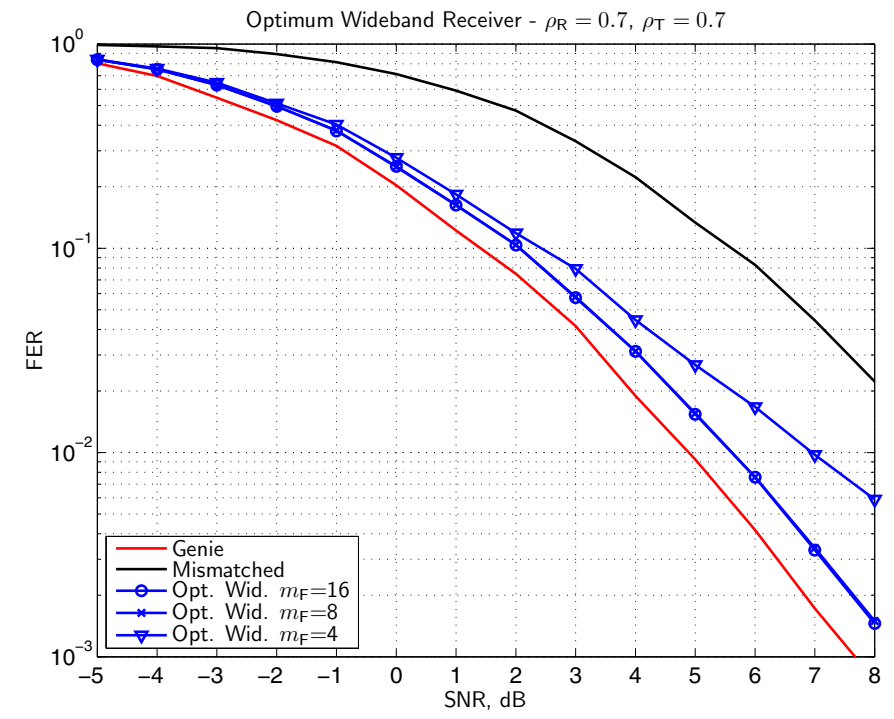

Fig. 3. FER vs. SNR of the MIMO-OFDM system with $\rho_{\mathrm{R}}=\rho_{\mathrm{T}}=0.7$. Genie, Mismatched and Optimum $\left(m_{\mathrm{F}}=4,8,16\right)$ receivers.

TABLE I

TIME COMPLEXITY OF DIFFERENT RECEIVER SCHEMES

\begin{tabular}{|c|c|c|}
\hline Receiver & & Time \\
\hline Mismatche & & 1 \\
\hline Optimum & $m_{\mathrm{F}}=4$ & 1.27 \\
\hline Optimum & $m_{\mathrm{F}}=8$ & 1.60 \\
\hline Optimum & $m_{\mathrm{F}}=16$ & 3.38 \\
\hline Optimum & no approximation & 153 \\
\hline
\end{tabular}

with the frequency correlation model of (18), it is enough to consider $m_{\mathrm{F}}=8$ eigenvalues to obtain the best performance of the Optimum receiver, whereas with $m_{\mathrm{F}}=4$ the penalty with the Genie receiver grows with SNR. To show the impact of $m_{\mathrm{F}}$ from the complexity point of view, we record in Tab. I the relative computing times required to decode one frame over an Intel ${ }^{\circledR}$ Xeon ${ }^{\mathrm{TM}} 3.20 \mathrm{GHz}$ computer with the receivers considered in this work.

\section{CONCLusions}

This work addressed the impact of imperfect channel state estimation over a MIMO-OFDM communication link affected by spatially correlated Rician fading. We consider a communication scenario compliant with the IEEE $802.11 \mathrm{n}$ assumptions. As a matter of fact, the receiver considered here can be regarded as extensions of previous receivers designed for the narrowband channel [1], [2] with uncorrelated Rayleigh fading and spatially correlated Rician fading. In this work we assume that the MIMO channel is broadband spatially and frequency correlated Rician fading. We extended the spatial Kronecker model to the frequency domain, and we designed an optimum receiver that processes jointly the pilot and data matrices to decode the transmitted symbol. Then, we simulated a MIMOOFDM system compliant with the IEEE 802.11n $2.0 \mathrm{draft}$ standard, and showed that a substantial power gain (more than $3 \mathrm{~dB}$ in the cases considered) is available with respect to the standard mismatched receiver. Finally, it is worth noting that the relative increase in time complexity entailed by the optimum receiver proposed with respect to the mismatched receiver is about $60 \%$.

\section{APPENDIX}

\section{A. Identities}

From [7] we know that: for any matrices $\mathbf{A}, \mathbf{B}, \mathbf{X}, \mathbf{Y}$ such that $\mathbf{A} \mathbf{X}^{\mathrm{H}} \mathbf{B Y}$ exists,

$$
\operatorname{Tr}\left(\mathbf{A X} \mathbf{X}^{\mathrm{H}} \mathbf{B Y}\right)=\operatorname{vec}\{\mathbf{X}\}^{\mathrm{H}}\left(\mathbf{A}^{\boldsymbol{\top}} \otimes \mathbf{B}\right) \operatorname{vec}\{\mathbf{Y}\}
$$

In [2] the following theorem was derived:

Theorem 1: Let $\mathbf{H}$ be an $m \times n$ matrix of independent circularly-symmetric zero-mean complex Gaussian random variables with unit variance. Let $\mathbf{A}$ and $\mathbf{R}$ be Hermitian matrices of dimensions $n \times n$ and $m \times m$, respectively, and $\mathbf{B}$ be any $m \times n$ matrix. Then, the following identity holds:

$$
\begin{aligned}
& \mathbb{E}_{\mathbf{H}}\left[\operatorname{etr}\left(-\left(\mathbf{H} \mathbf{A} \mathbf{H}^{\mathrm{H}} \mathbf{R}+\mathbf{H B}^{\mathrm{H}}+\mathbf{B H}^{\mathrm{H}}\right)\right)\right] \\
& =\operatorname{det}\left(\mathbf{I}_{m n}+\mathbf{C}\right)^{-1} \exp \left(\operatorname{vec}\{\mathbf{B}\}^{\mathrm{H}}\left(\mathbf{I}_{m n}+\mathbf{C}\right)^{-1} \operatorname{vec}\{\mathbf{B}\}\right)
\end{aligned}
$$

where $\mathbf{C} \triangleq \mathbf{A}^{\top} \otimes \mathbf{R}$

\section{REFERENCES}

[1] G. Taricco and E. Biglieri, "Space-time decoding with imperfect channel estimation," Wireless Communications, IEEE Transactions on, vol. 4, no. 4, pp. 1874-1888, 2005.

[2] G. Taricco and G. Coluccia, "Optimum Receiver Design for Correlated Rician Fading MIMO Channels with Pilot-Aided Detection," Selected Areas in Communications, IEEE Journal on, vol. 25, no. 7, pp. 1311$1321,2007$.

[3] J. Kermoal, L. Schumacher, K. Pedersen, P. Mogensen, and F. Frederiksen, "A stochastic MIMO radio channel model with experimental validation," Selected Areas in Communications, IEEE Journal on, vol. 20, no. 6, pp. 1211-1226, 2002.

[4] H. Ozcelik, M. Herdin, W. Weichselberger, J. Wallace, and E. Bonek, "Deficiencies of'Kronecker'MIMO radio channel model," Electronics Letters, vol. 39, no. 16, pp. 1209-1210, 2003.

[5] G. Coluccia, G. Taricco, and C. Mecklenbrauker, "Performance of an optimum receiver scheme based on pilot-symbol channel estimation over a measured MIMO channel," Signal Processing Advances in Wireless Communications, 2007. SPAWC 2007. IEEE 8th Workshop on, pp. 1-5, 2007.

[6] Wireless LAN Medium Access Control (MAC) and Physical Layer (PHY) specifications, IEEE 802.11n Draft STANDARD for Information Technology, 2007.

[7] R. Horn and C. Johnson, Matrix Analysis. Cambridge University Press, 1985.

[8] H. Bolcskei, M. Borgmann, and A. Paulraj, "Impact of the propagation environment on the performance of space-frequency coded MIMOOFDM," Selected Areas in Communications, IEEE Journal on, vol. 21, no. 3, pp. 427-439, 2003.

[9] S. Alamouti, "A simple transmit diversity technique for wireless communications," Selected Areas in Communications, IEEE Journal on, vol. 16, no. 8, pp. 1451-1458, 1998.

[10] S. Loyka, "Channel capacity of MIMO architecture using the exponentialcorrelation matrix," Communications Letters, IEEE, vol. 5, no. 9, pp. 369-371, 2001.

[11] L. Fang and L. Milstein, "Successive interference cancellation in multicarrier DS/CDMA," Communications, IEEE Transactions on, vol. 48, no. 9 , pp. 1530-1540, 2000. 